\title{
KH 15D: A SPECTROSCOPIC BINARY ${ }^{1}$
}

\author{
John Asher Johnson, ${ }^{2}$ Geoffrey W. Marcy, ${ }^{2}$ Catrina M. Hamilton, ${ }^{3,4}{ }^{4}$ William Herbst, ${ }^{5}$ and Christopher M. Johns-Krull ${ }^{3,4}$ \\ Received 2004 March 3; accepted 2004 May 19
}

\begin{abstract}
We present the results of a high-resolution spectroscopic monitoring program of the eclipsing pre-mainsequence star KH 15D that reveal it to be a single-lined spectroscopic binary. We find that the best-fit Keplerian model has a period $P=48.38$ days, which is nearly identical to the photometric period. Thus, we find the best explanation for the periodic dimming of KH $15 \mathrm{D}$ is that the binary motion carries the currently visible star alternately above and behind the edge of an obscuring cloud. The data are consistent with the models involving an inclined circumstellar disk, as recently proposed by Winn et al. and Chiang \& Murray-Clay. We show that the mass ratio expected from models of pre-main-sequence evolution, together with the mass constraints for the visible star, restrict the orbital eccentricity to $0.68 \leq e \leq 0.80$ and the mass function to $0.125 M_{\odot} \leq$ $F_{M} / \sin ^{3} i \leq 0.5 M_{\odot}$.
\end{abstract}

Key words: binaries: spectroscopic — circumstellar matter — stars: individual (KH 15D) stars: pre-main-sequence — techniques: radial velocities

\section{INTRODUCTION}

$\mathrm{KH} 15 \mathrm{D}$ is a $\mathrm{K} 6-\mathrm{K} 7$ pre-main-sequence star that exhibits dramatic photometric variability (Kearns \& Herbst 1998). Every 48.35 days, the star's brightness dims by 3.5 mag and remains in this faint state for nearly half of the photometric period. These deep brightness minima are accompanied by a slight blueing of the star's color indexes (Herbst et al. 2002, hereafter $\mathrm{He} 02$ ), little or no change in spectral type (Hamilton et al. 2001), and an increase in linear polarization (Agol et al. 2004). This implies that the star is completely eclipsed by an optically thick, extended collection of dust grains, possibly in the form of a circumstellar disk. If this is the case, the serendipitous alignment of the KH 15D star-disk system may provide insights into the evolution of young stars and their interactions with their circumstellar environments.

While KH $15 \mathrm{D}$ has periodic eclipses, it cannot be an ordinary eclipsing binary, because of the long duration of minimum light. Recent theories postulate that there is, nonetheless, a currently unseen binary companion to the visible star. In a study of archival photographic plates, Johnson \& Winn (2004, hereafter JW04) discovered that the historical light curve of $\mathrm{KH} 15 \mathrm{D}$ is similar to the modern light curve but appears to be diluted by light from a second star. Motivated by these findings, Winn et al. (2004, hereafter W04) constructed a model composed of a binary system with the orbital plane inclined with respect to the edge of an optically thick screen. As the two stars orbit one another, the reflex motion carries one star

\footnotetext{
${ }^{1}$ Based on observations obtained at the W. M. Keck Observatory, which is operated as a scientific partnership among the California Institute of Technology, the University of California, and the Naional Aeronautics and Space Administration; at Las Campanas Observatory of the Carnegie Institution with the Magellan II Clay Telescope; and at McDonald Observatory of the University of Texas at Austin.

2 Department of Astronomy, University of California at Berkeley, Berkeley, CA 94720-3411; johnjohn@astron.berkeley.edu, gmarcy@astron.berkeley.edu.

${ }^{3}$ Visiting Astronomer, McDonald Observatory, which is operated by the University of Texas at Austin.

4 Department of Physics and Astronomy, Rice University, 6100 Main Street, Houston, TX 77005; cmham@rice.edu, cmj@rice.edu.

5 Van Vleck Observatory, Wesleyan University, Middletown, CT 06459; wherbst@wesleyan.edu.
}

alternately above and behind the edge of an opaque screen, causing the eclipses. The long-term evolution of the light curve is reproduced by allowing the screen to move slowly across the binary orbit - a feature of the model that led the authors to envision the screen as a precessing, circumbinary disk. A similar model is proposed by Chiang \& Murray-Clay (2004, hereafter CM04), who also envision the opaque screen as an inclined, precessing circumbinary disk, or "ring," with an inner edge truncated by tidal interactions with the binary and an outer edge possibly shepherded by a planetary companion. Both models provide explanations of the unique features of KH 15D's light curve. The W04 model makes quantitative predictions about the orbital parameters of the binary system, while the CM04 model provides a physical description of the circumbinary ring.

A fundamental question that has not yet been answered is whether $\mathrm{KH} 15 \mathrm{D}$ is a single or multiple stellar system. He02 (see also Hamilton et al. 2003, hereafter Ha03) first searched for evidence of orbital companions using high-resolution VLT spectra and reported a radial velocity change of $+3.3 \pm$ $0.6 \mathrm{~km} \mathrm{~s}^{-1}$ over two widely spaced epochs. However, one of the measurements was made during egress, when there was a strong possibility of contamination from scattered light or line profile distortions from a sharp-edged cloud only partially occulting the stellar disk.

Over the past 2 years, we have conducted a high-resolution, multisite spectroscopic monitoring campaign to determine whether or not KH 15D exhibits orbital motion indicative of a multiple system. Here we present the results of our study, which show that KH 15D undergoes significant radial velocity variations. The variations are consistent with a binary companion with an orbital period equal to the 48 day photometric period. In $\S 2$, we summarize our observations and reduction procedures. The radial velocity measurements and best-fit Keplerian orbital parameters are presented in $\S 3$. We conclude in $\S 4$ with a discussion of our findings and implications for existing models of the KH 15D eclipse mechanism.

\section{DATA}

During the 2002-2003 and 2003-2004 observing seasons, we made 16 observations of $\mathrm{KH} 15 \mathrm{D}$ at maximum light, 
TABLE 1

Spectroscopic Observations of KH 15D

\begin{tabular}{|c|c|c|c|c|c|}
\hline UT Date & $\begin{array}{l}\text { Telescope and } \\
\text { Instrument }\end{array}$ & $\begin{array}{l}\Delta \lambda \\
(\AA)\end{array}$ & $R$ & $\begin{array}{c}\text { JD } \\
(2,400,000+)\end{array}$ & $\begin{array}{c}v_{r} \\
\left(\mathrm{~km} \mathrm{~s}^{-1}\right)\end{array}$ \\
\hline 2002 Oct $25 \ldots \ldots \ldots$ & Keck/HIRES & $3900-6200$ & 70,000 & $52,572.574$ & $1.7 \pm 0.2^{\mathrm{a}}$ \\
\hline 2002 Oct $28 \ldots \ldots \ldots$ & Keck/HIRES & $3900-6200$ & 70,000 & $52,575.511$ & $3.0 \pm 0.3^{\mathrm{a}}$ \\
\hline 2002 Dec $16 \ldots \ldots$. & Magellan/MIKE & $4500-6800$ & 45,000 & $52,624.738$ & $3.1 \pm 0.2^{\mathrm{b}}$ \\
\hline 2003 Jan $14 \ldots \ldots \ldots . .$. & Keck/HIRES & $3900-6200$ & 70,000 & $52,653.468$ & $3.3 \pm 0.2^{\mathrm{a}}$ \\
\hline 2003 Feb $8 \ldots \ldots \ldots . . .$. & Keck/HIRES & $4200-6600$ & 70,000 & $52,678.400$ & $9.0 \pm 0.5^{\mathrm{b}}$ \\
\hline 2003 Feb $9 \ldots \ldots \ldots \ldots$ & Keck/HIRES & $4200-6600$ & 70,000 & $52,679.410$ & $11.5 \pm 0.5^{\mathrm{b}}$ \\
\hline 2003 Mar $9 . . . . . . . . . .$. & Magellan/MIKE & $4900-8700$ & 22,000 & $52,707.511$ & $0.8 \pm 0.2^{\mathrm{b}}$ \\
\hline 2003 Nov $3 \ldots \ldots \ldots . . .$. & Keck/HIRES & $4200-6600$ & 30,000 & $52,946.584$ & $1.2 \pm 0.4^{\mathrm{b}}$ \\
\hline 2003 Nov $4 \ldots \ldots \ldots$ & Keck/HIRES & $4200-6600$ & 30,000 & $52,947.594$ & $1.8 \pm 0.3^{\mathrm{b}}$ \\
\hline 2004 Jan $4 \ldots \ldots \ldots \ldots . . .$. & McDonald/CE & $5600-6900$ & 30,000 & $53,008.762$ & $1.4 \pm 0.6^{\mathrm{b}}$ \\
\hline 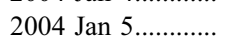 & McDonald/CE & $5600-6900$ & 30,000 & $53,009.852$ & $1.7 \pm 0.4^{\mathrm{b}}$ \\
\hline \multirow[t]{2}{*}{2004 Jan $10 \ldots \ldots \ldots$} & Keck/HIRES & $3900-6200$ & 70,000 & $53,014.320$ & $5.4 \pm 0.3^{\mathrm{a}}$ \\
\hline & McDonald/CE & $5600-6900$ & 30,000 & $53,014.760$ & $5.8 \pm 0.4^{\mathrm{b}}$ \\
\hline 2004 Jan $11 \ldots \ldots \ldots . . .$. & Keck/HIRES & $3900-6200$ & 70,000 & $53,015.506$ & $7.0 \pm 0.2^{b}$ \\
\hline 2004 Feb $9 \ldots \ldots \ldots \ldots$ & Keck/HIRES & $3900-6200$ & 70,000 & $53,044.834$ & $1.8 \pm 0.2^{\mathrm{a}}$ \\
\hline 2004 Feb $10 \ldots \ldots \ldots$ & Keck/HIRES & $3900-6200$ & 70,000 & $53,045.828$ & $1.3 \pm 0.2^{\mathrm{a}}$ \\
\hline
\end{tabular}

${ }^{a}$ Multiple reference stars were used to calculate the radial velocity. The reported velocity is the mean velocity measured from the various target-reference star pairs. The estimated uncertainty is the standard deviation of the mean velocity. See Table 2 for a listing of individual reference stars and radial velocity measurements for each target-reference star pair.

b Only one reference star was used in the radial velocity measurement. The reported velocity is the mean velocity from all echelle orders. The estimated uncertainty is the standard deviation of the mean velocity.

including 11 spectra with the $10 \mathrm{~m}$ Keck I Telescope and HIRES echelle spectrometer, two spectra with the $6.5 \mathrm{~m}$ Magellan II (Clay) telescope and the MIKE echelle spectrometer, and three spectra with the $2.1 \mathrm{~m}$ Otto Struve Telescope at McDonald Observatory with the Sandiford Cassegrain Echelle Spectrometer (CE). The observations are summarized in Table 1. Additional Keck HIRES spectra were obtained during minimum light. However, since it is unlikely that the star's photosphere is visible through the obscuring material, we decided to exclude from our analysis spectra that were obtained within 10 days of mid-eclipse. In addition to our own measurements, we also include the out-of-eclipse radial velocity measurement, $v_{r}=9.0 \pm 0.2 \mathrm{~km} \mathrm{~s}^{-1}$, reported by $\mathrm{Ha} 03$ based on their observations with the VLT UV-Visual Echelle Spectrograph.

Most of the HIRES observations were made as part of the California and Carnegie Planet Search. ${ }^{6}$ For these observations, the relatively faint apparent magnitude of $\mathrm{KH} 15 \mathrm{D}(V=16)$ at maximum light precluded the use of the iodine cell to establish a wavelength scale. The cell was therefore removed from the light path during observations of $\mathrm{KH} 15 \mathrm{D}$ to increase the throughput of the spectrometer. The raw CCD frames from all telescopes were reduced using reduction packages written in IDL. The details of the reduction procedures are fundamentally identical to the algorithm presented by Valenti (1994). After bias subtraction, each echelle frame is divided by a normalized median flat-field image. Order definition is performed using a bright star or flat-field exposure, and scattered light is removed by fitting a two-dimensional B-spline to the interorder regions and interpolating across each spectral order. After the scattered light is subtracted, each order is rectified, sky-subtracted, and summed in the cross-dispersion direction to form the final one-dimensional spectrum.

Instead of a summation in the cross-dispersion direction, the rectified orders of the McDonald spectra are reduced to

\footnotetext{
${ }^{6}$ See http://www.exoplanets.org.
}

one-dimensional spectra using the optimal extraction algorithm described by Hinkle et al. (2000). In the case of the MIKE reductions, the standard code is modified to correct for the tilt of the spectrometer entrance slit with respect to the CCD columns. The correction of the slit tilt is necessary because the sky subtraction algorithm we employ requires that the projected slit image lie parallel to the detector columns.

The radial velocity of $\mathrm{KH} 15 \mathrm{D}$ relative to the solar system barycenter is measured from each spectral observation by means of a cross-correlation analysis. For the spectra of $\mathrm{KH}$ 15D obtained as part of the Planet Search observing program, the program stars observed on each night provided an extensive selection of reference stars with known barycentric radial velocities, listed in Nidever et al. (2002). We selected reference stars that were observed within 30 minutes of KH 15D and with spectral types ranging from M0 to G5. Since the Planet Search target stars are observed through an iodine cell, orders containing iodine absorption lines are avoided in the analysis. For KH 15D observations obtained as part of programs other than the Planet Search, a single K-type reference star observed either before or after KH 15D is used as the reference star.

The cross-correlation of each $\mathrm{KH} 15 \mathrm{D}$ spectrum with respect to the reference spectra is performed using custom routines written in IDL. The procedure involves first rebinning each one-dimensional spectral order onto a new wavelength scale that is linear in $\log \lambda$. This ensures that each pixel in the rebinned spectrum represents a velocity interval that is uniform over the entire spectral order (Tonry \& Davis 1979). Regions containing telluric lines, strong emission features, and CCD defects are masked out, and each spectral order is cross-correlated with respect to the corresponding order of the reference spectrum. Each spectral order thus yields an independent measurement of the radial velocity of KH 15D relative to the reference star. The average of the ensemble set of velocities from all orders is then adopted as the relative radial velocity of KH 15D for a given epoch. 
TABLE 2

Absolute Radial Velocity Measurements of KH 15D from Each Reference Star

\begin{tabular}{|c|c|c|}
\hline $\begin{array}{c}\text { JD } \\
(2,400,000+)\end{array}$ & $\begin{array}{c}\text { Reference Star } \\
\text { HD No. }\end{array}$ & $\begin{array}{c}v_{r}^{\mathrm{a}} \\
\left(\mathrm{km} \mathrm{s}^{-1}\right)\end{array}$ \\
\hline \multirow{5}{*}{$52,572.574 \ldots \ldots$} & 40979 & $1.6 \pm 0.1$ \\
\hline & 56274 & $2.1 \pm 0.1$ \\
\hline & 52265 & $2.1 \pm 0.08$ \\
\hline & 49674 & $1.3 \pm 0.09$ \\
\hline & 66171 & $1.2 \pm 0.2$ \\
\hline \multirow[t]{5}{*}{$52,575.511 \ldots \ldots$} & 31966 & $2.9 \pm 0.2$ \\
\hline & 36395 & $4.0 \pm 0.08$ \\
\hline & 47157 & $3.2 \pm 0.2$ \\
\hline & 37124 & $2.2 \pm 0.1$ \\
\hline & 40979 & $2.7 \pm 0.3$ \\
\hline $52,624.738 \ldots \ldots$ & 10700 & $3.1 \pm 0.2$ \\
\hline \multirow[t]{5}{*}{$52,653.468 \ldots \ldots$} & 31966 & $3.6 \pm 0.1$ \\
\hline & 42618 & $2.7 \pm 0.1$ \\
\hline & 47157 & $3.1 \pm 0.07$ \\
\hline & 99109 & $3.6 \pm 0.09$ \\
\hline & 99492 & $3.4 \pm 0.1$ \\
\hline $52,678.400 \ldots \ldots$ & 36006 & $9.0 \pm 0.5$ \\
\hline $52,679.410 \ldots \ldots$ & 36006 & $11.5 \pm 0.5$ \\
\hline $52,707.511 \ldots \ldots$ & 36006 & $0.8 \pm 0.2$ \\
\hline $52,946.584 \ldots \ldots$ & 36006 & $1.2 \pm 0.4$ \\
\hline $52,947.594 \ldots \ldots$ & 36006 & $1.8 \pm 0.3$ \\
\hline $53,008.762 \ldots \ldots$ & 36006 & $1.4 \pm 0.6$ \\
\hline $53,009.852 \ldots \ldots$ & 17660 & $1.7 \pm 0.4$ \\
\hline \multirow[t]{2}{*}{$53,014.320 \ldots \ldots$} & 45350 & $5.1 \pm 0.2$ \\
\hline & 18830 & $5.6 \pm 0.3$ \\
\hline $53,014.760 \ldots \ldots$ & 17660 & $5.8 \pm 0.4$ \\
\hline $53,015.506 \ldots \ldots$ & 45350 & $7.0 \pm 0.2$ \\
\hline \multirow[t]{4}{*}{$53,044.834 \ldots \ldots$} & 50499 & $2.0 \pm 0.2$ \\
\hline & 46375 & $1.7 \pm 0.1$ \\
\hline & 50281 & $1.8 \pm 0.2$ \\
\hline & 46375 & $1.9 \pm 0.2$ \\
\hline \multirow[t]{4}{*}{$53,045.828 \ldots \ldots$} & 50499 & $1.6 \pm 0.1$ \\
\hline & 76909 & $1.3 \pm 0.1$ \\
\hline & 73667 & $0.9 \pm 0.1$ \\
\hline & 72673 & $1.2 \pm 0.1$ \\
\hline
\end{tabular}

${ }^{a}$ Velocities are the mean value from all echelle orders used in the cross-correlation analysis. Estimated uncertainties are the standard deviation of the mean velocity from all echelle orders.

The relative radial velocities from each night are converted into absolute barycentric radial velocities using the relation

$$
v_{\text {rad }}=\Delta v+\left(\mathrm{BC}_{\mathrm{kh}}-\mathrm{BC}_{\mathrm{ref}}\right)+v_{\mathrm{ref}} .
$$

In equation (1), $\Delta v$ is the relative velocity from the crosscorrelation analysis; $\mathrm{BC}_{\mathrm{kh}}$ and $\mathrm{BC}_{\mathrm{ref}}$ are the barycentric corrections for $\mathrm{KH} 15 \mathrm{D}$ and the reference star, respectively; and $v_{\text {ref }}$ is the absolute barycentric radial velocity of the reference star as listed in Nidever et al. (2002). The mean measured velocities from each epoch are listed in the final column of Table 1. Table 2 lists the velocities obtained from our crosscorrelation analysis for each individual reference star on each night.

For nights when only one reference spectrum is available, the uncertainty is estimated using the standard deviation of the mean radial velocity measured from all echelle orders. For nights with multiple reference spectra, the standard deviation of the velocities computed from each target-reference pair is adopted as the uncertainty. This latter method of error estimation typically yields a larger value than the order-toorder scatter seen for an individual observation $\left(0.2 \mathrm{~km} \mathrm{~s}^{-1} \leq\right.$ $\sigma_{v} \leq 0.6 \mathrm{~km} \mathrm{~s}^{-1}$ compared with $\left.0.1 \leq \sigma_{v} \leq 0.3\right)$. The larger scatter in velocity measurements among the target-reference star combinations is likely due to external systematic effects such as small shifts in the position of the CCD throughout the night caused by thermal relaxation of the detector mount. Thus, the standard deviation measured from the echelle orders in a single observation likely underestimates the true uncertainty by approximately a factor of 2 .

\section{RESULTS}

Figure 1 illustrates how the radial velocity of KH 15D varies temporally out of eclipse over a range of $10.7 \mathrm{~km} \mathrm{~s}^{-1}$. These data indicate that there must be an unseen star in the system, as had previously been inferred from the analysis of the historical light curve (JW04). Hereafter we will adopt the naming convention of W04 and refer to the currently visible star as $\mathrm{A}$ and the hidden companion as B.

\subsection{Orbit Solution}

Using a nonlinear least-squares algorithm, we found a bestfit model orbit with a period $P=48.38$ days, eccentricity $e \geq 0.27$, and velocity semiamplitude $K \geq 8.15 \mathrm{~km} \mathrm{~s}^{-1}$. The other orbit parameters are listed in Table 3 . Figure 2 shows a plot of radial velocity versus orbital phase for $P=48.38$ days. The rms scatter of the fit residuals is $0.38 \mathrm{~km} \mathrm{~s}^{-1}$ and the reduced $\sqrt{\chi_{\nu}^{2}}=1.5$. The vertical lines at $\phi=0.33$ and $\phi=0.73$ denote the approximate phases of ingress and egress, respectively, based on the $\mathrm{He} 02$ ephemeris. We have no radial velocity measurements between these phases because of the eclipse of star A.

We find that the orbit solution is not well constrained as a consequence of the lack of data near periapse, which allows the velocity semiamplitude of the orbit solution to compensate for changes in the eccentricity. As such, we were able to obtain reasonable fits by fixing the eccentricity at values $e>$ 0.27 , with each solution yielding different values of the orbit parameters and values of $\sqrt{\chi_{\nu}^{2}}$ that are equivalent at the $97.5 \%$ confidence level (based on the 9 degrees of freedom in the fit).

Figure 3 shows how $\sqrt{\chi_{\nu}^{2}}$ and the orbit parameters vary as a function of eccentricity. While the period varies little over a wide range of eccentricities, it is apparent that the radial velocity data alone provide poor constraints for the other orbit parameters. However, the implied mass ratio from other KH 15D observations, together with the measured mass limits of star A, can be used to place limits on the orbital eccentricity, as we now show.

\subsection{Orbit Parameter Constraints}

JW04 determined from photometric measurements of archival plates that the out-of-eclipse magnitude of KH 15D was 0.9 mag brighter (at $I$ band) 40 years ago compared with the modern bright state. The two most probable explanations for the brighter apparent magnitude in the past are that either both stars were visible or star $\mathrm{B}$ alone was visible. If both stars were visible, then $L_{B} / L_{A}=1.3$. If only B were visible, then $L_{B} / L_{A}=2.3$. In either case, the condition $L_{B} / L_{A}>1$ must hold.

Multicolor photometric measurements obtained during minimum light show a slight blueing of the color indexes (Ha03) compared with the colors at maximum light. Similarly, Agol et al. (2004) measure slightly bluer colors during eclipse 


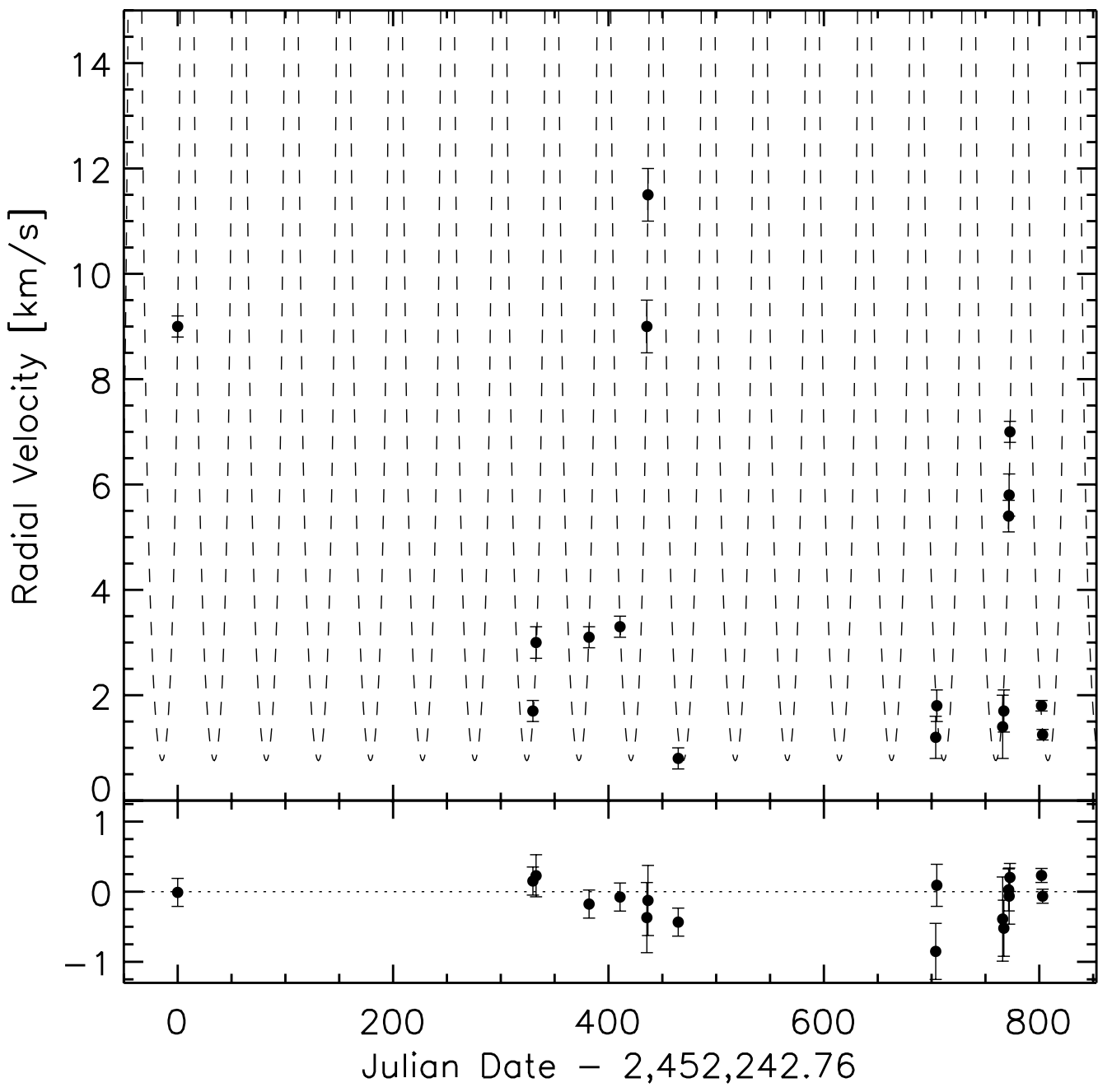

FIG. 1.-Radial velocity of KH $15 \mathrm{D}$ as a function of time. The dashed curve is the best-fit Keplerian orbit with the eccentricity fixed at $e=0.74$, the mean value allowed by our orbit constraints (see $\S 3.2$ ).

from their low-resolution spectropolarimetric observations compared with their out-of-eclipse observations. One possibility for the bluer color indexes during eclipse is that the scattered light is dominated by Rayleigh scattering. However, $\mathrm{He} 02$ show that there is no reddening of the light from star A during ingress and egress. This suggests that the opacity of the occulting material is wavelength independent and that the bluer colors are due to a bluer object. Therefore the temperature of B must be hotter than the temperature of A, assuming both stars contribute nearly equally to the scattered component

TABLE 3

Optimized Model Parameters

\begin{tabular}{|c|c|c|c|}
\hline Parameter & $\begin{array}{l}\text { Acceptable Fit } \\
\text { to Velocities }\end{array}$ & $\begin{array}{l}\text { Acceptable Fit } \\
\text { with } e=0.74\end{array}$ & $\begin{array}{c}\text { W04 } \\
\text { Model } 1\end{array}$ \\
\hline 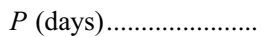 & 48.38 to 48.40 & $48.38(0.01)$ & 48.35 (fixed) \\
\hline 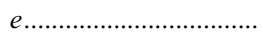 & $\geq 0.27$ & 0.74 (fixed) & 0.7 \\
\hline$\omega(\operatorname{deg}) \ldots \ldots \ldots \ldots \ldots \ldots \ldots$ & -20 to 1 & $-2(1)$ & -7.2 \\
\hline$\gamma^{\mathrm{b}}\left(\mathrm{km} \mathrm{s}^{-1}\right) \ldots \ldots \ldots \ldots \ldots$ & +6.9 to +22.5 & $+14.7(0.9)$ & +15.5 \\
\hline$a \sin i(\mathrm{AU}) \ldots \ldots \ldots \ldots$ & $\leq 0.29$ & $0.21(0.5)$ & 0.18 \\
\hline$K\left(\mathrm{~km} \mathrm{~s}^{-1}\right) \ldots \ldots \ldots \ldots \ldots$ & $\geq 8$ & $53(4)$ & 27.5 \\
\hline$F_{M} / \sin ^{3} i \ldots \ldots \ldots \ldots \ldots \ldots$ & $2.4 \times 10^{-3}$ to 2.2 & $0.24(0.05)$ & $3.8 \times 10^{-2}$ \\
\hline 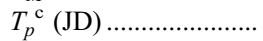 & $\ldots$ & $2,452,251.0(0.6)$ & $\ldots$ \\
\hline Fit rms $\left(\mathrm{km} \mathrm{s}^{-1}\right) \ldots \ldots \ldots$ & 0.38 & 0.38 & $\ldots$ \\
\hline Reduced $\sqrt{\chi_{\nu}^{2}} \ldots \ldots \ldots \ldots$ & 1.5 & 1.5 & $\ldots$ \\
\hline
\end{tabular}

a The limits on the fit parameters correspond to the $97.5 \%$ confidence upper limit on $\sqrt{\chi_{\nu}^{2}}$ (see Fig. 3) and the condition $e<1$.

$\mathrm{b}$ Radial velocity of the binary center of mass with respect to the solar system barycenter.

c Time of periastron passage. 


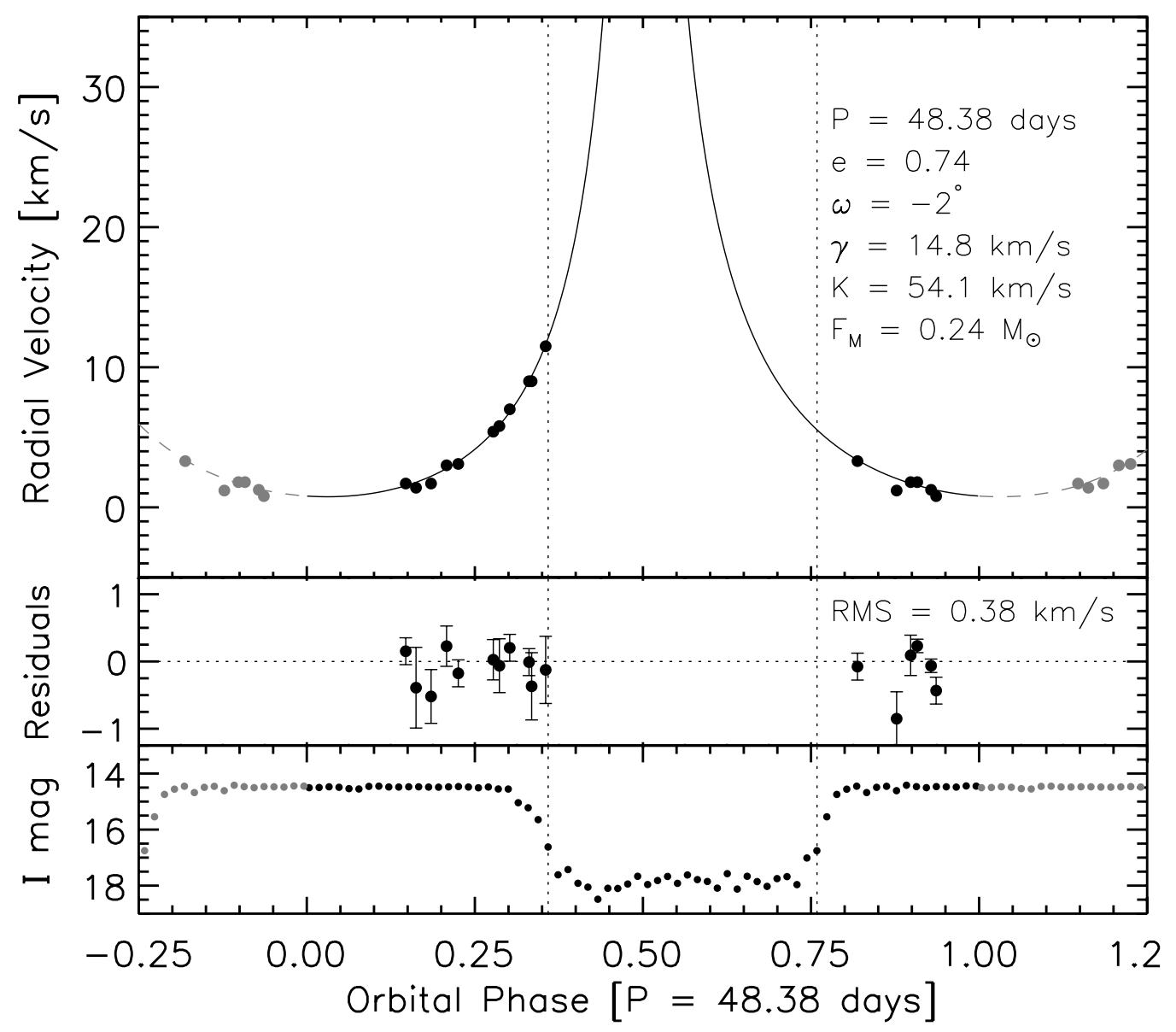

FIG. 2.-Radial velocity of KH 15D as a function of orbital phase. The solid curve is the best-fit Keplerian orbit with the eccentricity fixed at $e=0.74$, the mean value allowed by our orbit constraints (see $\S 3.2$ ). The fit has reduced chi-square $\sqrt{\chi_{\nu}^{2}}=1.5$. The vertical dotted lines represent the approximate phases of ingress (left) and egress (right) based on the He02 ephemeris. The bottom panel shows the 2001-2002 I-band light curve. The phased photometric measurements have been binned for clarity using phase intervals of $\Delta \phi=0.015$.

of the light observed during the eclipse of A. However, since the colors are only $\sim 0.1$ mag bluer at minimum light ( $\mathrm{He} 02$ ), $T_{B}$ cannot be much larger than $T_{A}$.

For most low-mass $\left(M_{*}<1.0 M_{\odot}\right)$ pre-main-sequence evolutionary models (e.g., Chabrier \& Baraffe 1997; D’Antona \& Mazzitelli 1997), the stellar mass is monotonic with both luminosity and temperature. For coeval stars on their Hayashi tracks, $T_{B} \gtrsim T_{A}$ and $L_{B} / L_{A}>1$ imply that $M_{B} \gtrsim M_{A}$ or, in terms of the mass ratio, $M_{A} / M_{B} \lesssim 1$.

The mass function of a Keplerian orbit can be expressed as

$$
F_{M}(e, K, P)=\frac{K^{3} P\left(1-e^{2}\right)^{3 / 2} \sin ^{3} i}{2 \pi G}=\frac{M_{B}^{3} \sin ^{3} i}{\left(M_{A}+M_{B}\right)^{2}},
$$

where $P$ is the orbital period and $K$ is the velocity semiamplitude. Solving equation (2) for $M_{A}$ yields

$$
M_{A}\left(e, M_{A} / M_{B}\right)=\frac{M_{A}}{M_{B}}\left(1+\frac{M_{A}}{M_{B}}\right)^{2}\left[\frac{F_{M}(e, K, P)}{\sin ^{3} i}\right] .
$$

The mass function $F_{M} / \sin ^{3} i$ in equation (3) is calculated from the parameters of the best-fit orbit to the radial velocity data with the value of $e$ fixed in the fitting procedure. Figure 4 shows plots of $M_{A}$ versus $e$ for $M_{A} / M_{B}=0.7,0.8,0.9$ and 1.0. Also shown is the value of $M_{A} / M_{B}=1.6$ obtained by W04 based on their model fit to the KH 15D light curve (see $\S 4$ ). Since the mass of the visible star is known to fall within the limits $0.5 M_{\odot} \leq M_{A} \leq 1.0 M_{\odot}$ (Flaccomio et al. 1999; Park et al. 2000), it can be seen by inspection of Figure 4 that acceptable choices of the eccentricity fall within the range $0.68 \leq e \leq 0.80$.

The resulting range of acceptable orbit parameter values is shown in Figure 3 between the solid vertical lines in each panel. The third column of Table 3 lists the best-fit parameter values obtained using the mean acceptable value of the eccentricity, $e=0.74$ (see also Fig. 2). The uncertainties in the fit parameters were estimated using a Monte Carlo simulation. We generated $10^{3}$ statistical realizations of the velocity time series assuming the errors are normally distributed with standard deviations equal to the measurement uncertainties. The estimated uncertainties are also displayed in Figure 3, as error bars on the points located at $e=0.74$.

\subsection{Periodicity}

As discussed in $\S 3.1$, we find the best-fit Keplerian has an orbital period of $P=48.38 \pm 0.01$ days. $\mathrm{He} 02$ report a period of $P=48.35 \pm 0.02$ days from their photometric monitoring, and JW04 report $P=48.42 \pm 0.02$ days from a periodogram analysis of archival photographic plates photometry. Thus, our measured orbital period differs by $1.5 \sigma$ and $2 \sigma$ from the modern and historical photometric periods, respectively. Since 


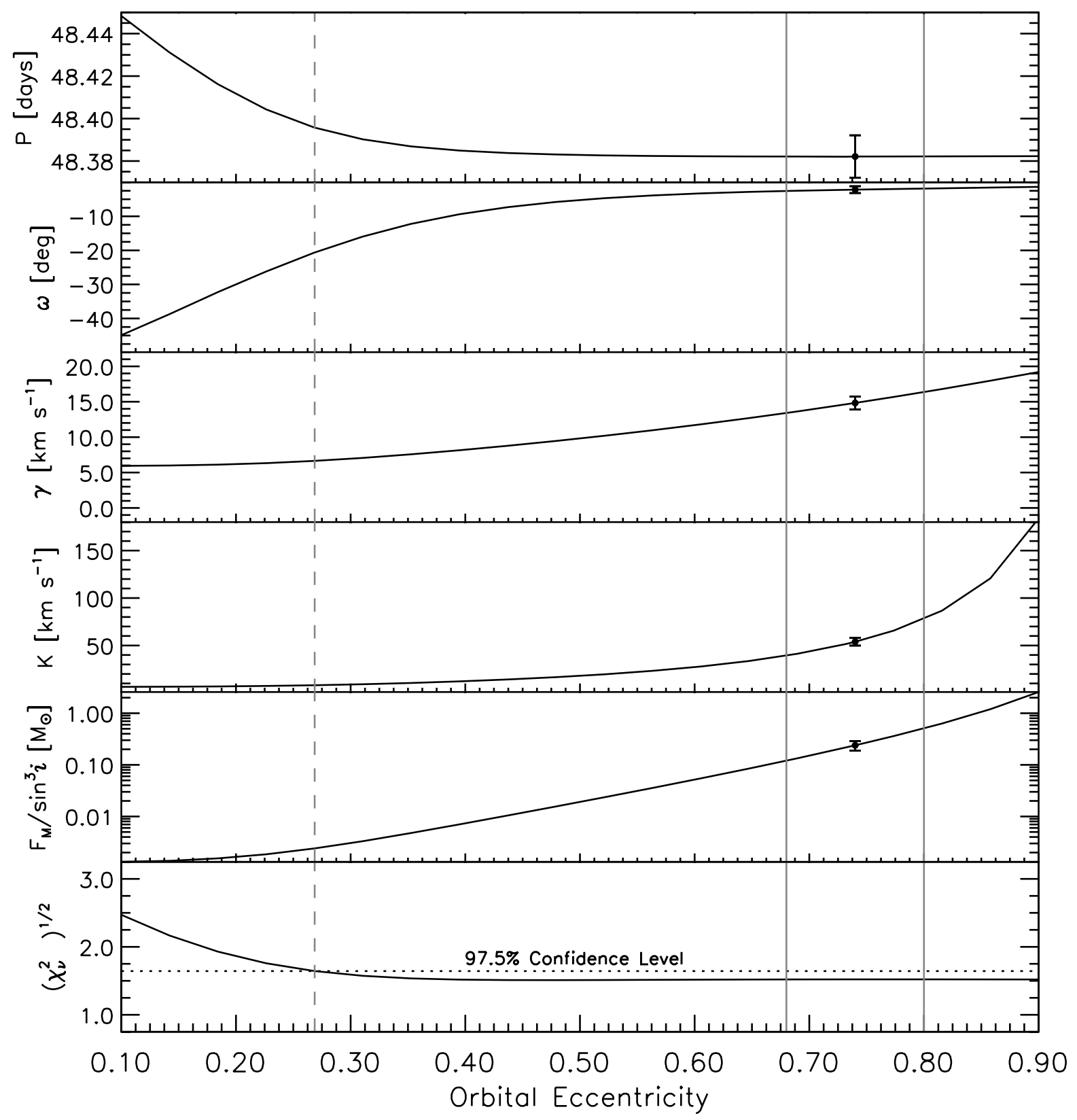

FIG. 3.-Dependence of the best-fit orbit parameters (top five panels) and reduced $\chi^{2}$ (bottom) on the orbital eccentricity. The ordinate values are generated by fixing the orbital eccentricity in the least-squares fit to the radial velocity data. The vertical dashed line indicates the lower limits placed on the fit parameters based on the $97.5 \%$ confidence level for $\sqrt{\chi_{\nu}^{2}}$ with 9 degrees of freedom. The solid vertical lines denote the upper and lower limits placed on the eccentricity as discussed in $\S 3.2$ (see also Fig. 4), and the fits generated for $e=0.74$ are denoted by circles with error bars.

the evolving shape of the light curve may complicate the accurate determination of the photometric period, we conclude that the orbital period and photometric period of $\mathrm{KH} 15 \mathrm{D}$ are indistinguishable within measurement errors.

$\mathrm{He} 02$ noted that the shape of the light curve varies from eclipse to eclipse in such a way as to suggest a 96 day period for the system, with each cycle containing two brightness minima. In order to investigate this alternative periodicity, we fixed the period in our fit at $P=96.76$ days and obtained a reduced $\sqrt{\chi_{\nu}^{2}}$ of 47.5 . Based on this poor quality of fit, we find that a 96 day period is incompatible with our radial velocity measurements.

\section{DISCUSSION}

The results of our spectroscopic monitoring campaign show that $\mathrm{KH} 15 \mathrm{D}$ is a spectroscopic binary system. The observed radial velocities are consonant with a stellar companion with an orbital period equal to the photometric period. We now discuss the implications of the binarity of KH 15D for current models of the photometric variability mechanism.

Existing models of the KH $15 \mathrm{D}$ eclipse mechanism fall into two classes differentiated by whether it is the orbital motion of the star or a feature of a circumstellar disk that causes the photometric variability. The first class of models posits the existence of a single star surrounded by a circumstellar disk containing a nonaxisymmetric density enhancement or, alternatively, a warp. As the disk feature orbits the star with a 48 day period, it periodically blocks the line of sight to the stellar surface. Based on their spectropolarimetric observations, which showed an increase in polarization during minimum light, Agol et al. (2004) developed a model of a warped disk with an extended atmosphere and obtained a reasonable 


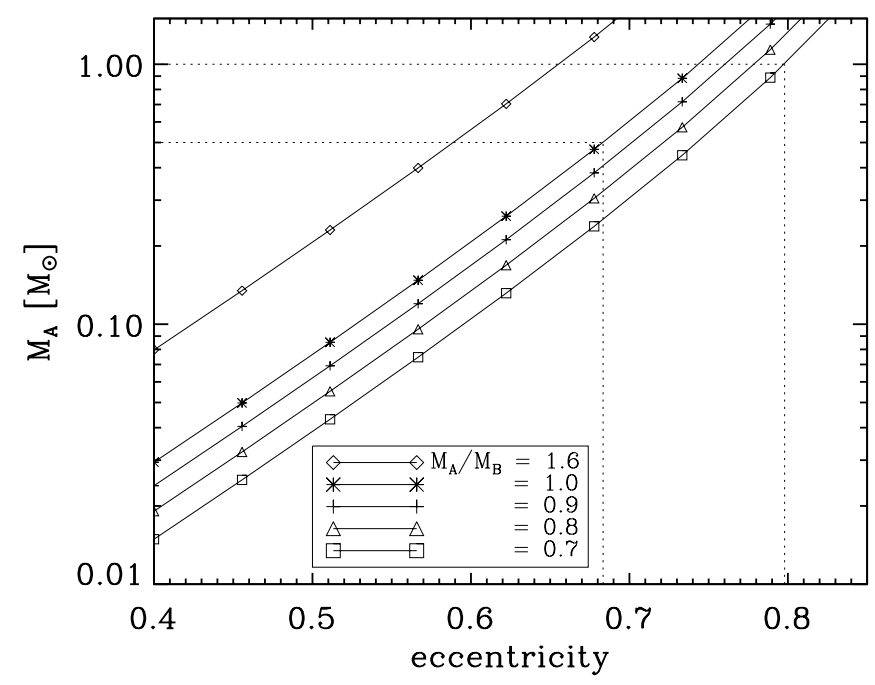

FIG. 4.-Mass of the visible component of $\mathrm{KH} 15 \mathrm{D}, M_{A}$, vs. orbital eccentricity for five choices of the mass ratio $M_{A} / M_{B}$. The dotted lines show that for $0.5 M_{\odot}<M_{A}<1.0 M_{\odot}$ and $M_{A} / M_{B} \lesssim 1.0$, acceptable values of the eccentricity fall within the range $0.68 \leq e \leq 0.80$. The inclination is assumed to be $i=90^{\circ}\left(\sin ^{3} i=0\right)$.

fit to the 2001-2002 light curve. A similar analysis was performed by Barge \& Viton (2003) using a model involving a large dusty vortex.

While these single-star models are able to match the light curve of $\mathrm{KH} 15 \mathrm{D}$ at one point in time, they do not adequately explain the temporal changes in the observed light curve. Perhaps more importantly, it is not clear if a binary companion to $\mathrm{KH} 15 \mathrm{D}$ is compatible with an eclipsing disk feature. If a density enhancement in a circumstellar disk is responsible for the 48 day photometric period, the feature must orbit the central star at a distance of $\sim 0.22 \mathrm{AU}$, assuming a Keplerian orbit. This geometry would restrict the stellar companion to orbit either at a distance less than $0.22 \mathrm{AU}$ or beyond the outer extent of the circumstellar disk. However, since our best-fit orbit yields a period of $48.38 \pm 0.01$ days and $a \sin i=0.21$ AU (assuming $e=0.74$; see $\S 3.2$ ) for the binary companion, neither of these scenarios seems plausible. Thus, it does not seem possible for both an eclipsing disk feature and a stellar-mass companion to coexist with the same orbital period.

In the second class of models, proposed by W04 and CM04, there exists an unseen binary companion of comparable mass to the visible K-type star seen today. Surrounding the two stars is a circumbinary disk viewed nearly edge-on. The orbital plane of the two stars is tilted at a small angle with respect to the disk plane and the present-day light curve is produced as the reflex motion of star A carries it above and behind the disk plane.

Independent evidence for the binary nature of KH 15D has emerged from studies of archival photographic plates. In a study of photographic plates obtained from Asiago Observatory, JW04 show that the apparent magnitude of KH 15D was variable from 1968 to 1983 but the light curve from this epoch was markedly different from the one observed today. The bright state was nearly a factor of 2 brighter in the past and the eclipse depth was a factor of 5 shallower. These findings can be explained by invoking the presence of a second star that was visible in the past but is unseen today (JW04).

Motivated by these findings, W04 constructed a model of an eccentric binary with a fraction of the orbital plane obscured by an opaque screen (or circumbinary disk) and found a quantitative orbital solution by fitting simultaneously to the
2001-2002 photometry of $\mathrm{He} 02$, the historical photometry of JW04 and the radial velocity measurements of $\mathrm{Ha} 03$. Based on a preliminary investigation of the radial velocities presented here, CM04 independently used physical arguments to propose a similar model of an eccentric binary surrounded by a nodally precessing circumbinary ring. As the ring precesses, the light curve gradually changes from the one recovered from the archival plates to the shape seen today. Perhaps the greatest advantage of the two-star models is their ability to explain not only the present-day light curve, but also its evolution over the past half-century.

In addition to explaining the photometric phenomenology of $\mathrm{KH} 15 \mathrm{D}$, the two-star models make predictions about the nature of the binary orbit. Both CM04 and W04 predict that (1) periastron passage occurs during minimum light, (2) the orbital companion has a mass comparable to the currently visible star, and (3) the binary orbit is highly eccentric. These predictions are precisely what we find from our orbit solution.

The W04 model makes additional, quantitative predictions about the orbital parameters of the binary. For an assumed fixed period of 48.35 days, the model produces a velocity semiamplitude of $27.5 \mathrm{~km} \mathrm{~s}^{-1}$, eccentricity $e=0.7$, a mass ratio $M_{A} / M_{B}=1.6$, argument of pericenter $\omega=-7^{\circ} .2$, and a center-of-mass radial velocity $\gamma=+15.5 \mathrm{~km} \mathrm{~s}^{-1}$. As can be seen in Table 3, the predictions of W04 model 1 agree well with our orbital solution with fixed eccentricity, $e=0.74$ (see $\S 3.2$ ). We note here that our center-of-mass velocity for the binary system of $\gamma=+14.7 \pm 0.9 \mathrm{~km} \mathrm{~s}^{-1}$ clearly rules out W04 model 2, which predicts $\gamma=+5.7 \mathrm{~km} \mathrm{~s}^{-1}$ and negative velocity at periapse.

The W04 model also predicts the inclination of the binary orbit, a property of the system that our radial velocities do not. W04 model 1 predicts $i=84^{\circ} .6$, or $\sin i=0.996$. Similarly, using the geometry of the circumbinary ring proposed by CM04, an upper limit on the inclination of $i<80^{\circ}$ can be assigned to the binary orbit based on the time lag between periastron passage and mid-eclipse (E. Chiang 2004, private communication).

We note that the W04 model has the peculiar feature that the less massive star is the more luminous. Using equation (3), the values of $P, K$, and $e$ produced by the W04 model lead to a mass function $F_{M}=0.038 M_{\odot}$ and $M_{A}=0.41 M_{\odot}$. This mass is significantly less than the lower limit of $0.5 M_{\odot}$ measured by Park et al. (2000) and the value of $0.6 M_{\odot}$ measured by Flaccomio et al. (1999). Figure 4 shows a plot of $M_{A}$ as a function of $e$ for the W04 mass ratio $M_{B} / M_{A}=1.6$. For our best-fitting model parameters, only eccentricities between 0.58 and 0.65 yield a mass of A between 0.5 and $1.0 M_{\odot}$. Therefore, for a mass ratio of 1.6, the eccentricity reported by W04 $(e=0.7)$ is larger than the value allowed by our radial velocity measurements, assuming $0.5 M_{\odot} \leq M_{A} \leq 1.0 M_{\odot}$. However, this discrepancy is not too surprising, since the W04 model used only two radial velocity measurements. It is also important to note that the mass limits on star A are derived by placing $\mathrm{KH} 15 \mathrm{D}$ on a theoretical $\mathrm{H}-\mathrm{R}$ diagram and are therefore subject to the accuracy of the pre-main-sequence evolutionary model employed. In all other features of the $\mathrm{KH} 15 \mathrm{D}$ binary system, there is a remarkable agreement between the W04 model and the orbital solution calculated from the radial velocities. Because of the strong evidence of a second star from our radial velocity measurements and the historical photometry, we find the two-star class of model to be the most compelling explanation of the $\mathrm{KH} \mathrm{15D}$ photometric variability mechanism. 
However, the case of the "winking star" is still far from closed. A key missing aspect of the two-star models is direct detection of a circumbinary disk around KH 15D. He02 report a lack of near-infrared excess and a null detection at millimeter wavelengths. CM04 state that such findings are consistent with a circumbinary ring having an inner radius of $\sim 1$ AU that is tidally truncated by the central binary, and an outer radius of $\sim 5 \mathrm{AU}$ that is possibly shepherded by an as yet unseen planet. They predict mid-infrared fluxes that are observable with the Spitzer Space Telescope. Clearly such observations will be vital in further development of models of the KH 15D system.
We would like to thank Gibor Basri, Paul Butler, Debra Fischer, and Subanjoy Mohanty for generously lending portions of their observing time for our project. Many thanks to Eugene Chiang, Ruth Murray-Clay, Steve Dawson, and Josh Winn for their helpful conversations and suggestions. We acknowledge support by NASA grant NAG 5-8299 and NSF grant AST 95-20443 (to G. W. M.), NASA grant NAG 5-12502 (to W. H.), and Sun Microsystems. We thank the NASA, University of California, and McDonald Observatory telescope assignment committees for allocations of telescope time.

\section{REFERENCES}

Agol, E., Barth, A. J., Wolf, S., \& Charbonneau, D. 2004, ApJ, 600, 781

Barge, P., \& Viton, M. 2003, ApJ, 593, L117

Chabrier, G., \& Baraffe, I. 1997, A\&A, 327, 1039

Chiang, E. I., \& Murray-Clay, R. A. 2004, ApJ, 607, 913 (CM04)

D’Antona, F., \& Mazzitelli, I. 1997, Mem. Soc. Astron. Italiana, 68, 807

Flaccomio, E., Micela, G., Sciortino, S., Favata, F., Corbally, C., \& Tomaney, A. 1999, A\&A, 345, 521

Hamilton, C. M., Herbst, W., Mundt, R., Bailer-Jones, C. A. L., \& Johns-Krull, C. M. 2003, ApJ, 591, L45 (Ha03)

Hamilton, C. M., Herbst, W., Shih, C., \& Ferro, A. J. 2001, ApJ, 554, L201

Herbst, W., et al. 2002, PASP, 114, 1167 ( $\mathrm{He} 02)$
Hinkle, K., Wallace, L., Valenti, J., \& Harmer, D. 2000, Visible and Near Infrared Atlas of the Arcturus Spectrum, 3727-9300 ̊̊ (San Francisco: ASP) Johnson, J. A., \& Winn, J. N. 2004, AJ, 127, 2344 (JW04)

Kearns, K. E., \& Herbst, W. H. 1998, AJ, 116, 261

Nidever, D. L., Marcy, G. W., Butler, R. P., Fischer, D. A., \& Vogt, S. S. 2002, ApJS, 141, 503

Park, B.-G., Sung, H., Bessell, M. S., \& Kang, Y. H. 2000, AJ, 120, 894

Tonry, J., \& Davis, M. 1979, AJ, 84, 1511

Valenti, J. A. 1994, Ph.D. thesis, Univ. California, Berkeley

Winn, J. N., Holman, M. J., Johnson, J. A., Stanek, K. Z., \& Garnavich, P. M. 2004, ApJ, 603, L45 (W04) 